\title{
A Mouse Model for Inflammatory Bowel Disease Associated Tumorigenesis
}

\author{
Zexian Chen, Yongle Chen, Xiaowen He, Ping Lan* \\ Department of Colorectal Surgery, the Sixth Affiliated Hospital, Sun Yat-sen University, Guangzhou, China \\ Email address: \\ chenzexian@foxmail.com (Zeixan Chen), zslychyle@163.com (Yongle Chen), hexiaowen7003@126.com (Xiaowen He), \\ sumslp@163.com (Ping Lan) \\ ${ }^{*}$ Corresponding author
}

To cite this article:

Zexian Chen, Yongle Chen, Xiaowen He, Ping Lan. A Mouse Model for Inflammatory Bowel Disease Associated Tumorigenesis. International Journal of Clinical and Experimental Medical Sciences. Vol. 4, No. 4, 2018, pp. 63-67. doi: 10.11648/j.ijcems.20180404.12

Received: September 6, 2018; Accepted: September 20, 2018; Published: October 12, 2018

\begin{abstract}
Inflammatory bowel disease, including Crohn's disease and ulcerative colitis, is one kind of chronic, relapsing inflammatory disorder of the gastrointestinal tract and has the potential of tumorigenesis. However, no stable and mature cell line is available for relative basic research, so animal model is essential. In this study, a mice model was built, which mimics the dynamic disease course of inflammatory bowel disease associated tumorigenesis with injection of azoxymethane and sequent cycles of drinking water with dextran sulfate sodium. All the mice survived till the end of the modeling procedure. Compared with the mice in the control group, the mice in the modeling group experienced obvious bloody diarrhea and body weight loss $(\mathrm{P}<0.001)$. The colorectum was significantly swollen and shorter $(\mathrm{P}<0.001)$ while the spleen was significantly bigger $(\mathrm{P}<0.001)$ in mice in the modeling group compared with the mice in the control group. All the mice in the modeling group developed tumors in the colorectum, with an average tumor number of 6.5 and an average tumor size of $10.25 \mathrm{~mm}^{3}$. Pathological evaluation by hematoxylin and eosin staining confirmed the tumors as adenomas with high-grade dysplasia. In conclusion, this model is inducible and stable which would be very useful in the research in this field.
\end{abstract}

Keywords: Inflammatory Bowel Disease, Tumorigenesis, Mice Model

\section{Introduction}

Inflammatory bowel disease (IBD) is one kind of chronic, relapsing inflammatory disorder of the gastrointestinal tract, which is represented mainly by ulcerative colitis and Crohn's disease [1]. Patients with IBD often have some kinds of complications such as intestinal obstruction, perforation and perianal lesions. Among all the complications of IBD, carcinogenesis should be the most serious [2, 3]. Adenocarcinoma of the small bowel is extremely rare, compared with adenocarcinoma of the colon and rectal. IBD contributes only $1-2 \%$ to all cases of colorectal cancer, but according to preliminary statistics, colorectal cancer accounts for approximately $15 \%$ of all deaths in patients with $\operatorname{IBD}[4,5]$. The risk of colorectal cancer for people with IBD increases by $0.5-1 \%$ yearly, $8-10$ years after diagnosis [6]. Eaden et al. found the overall incidence rate of colorectal cancer was $3 / 1000$ person years duration and cumulative risks of colorectal cancer in patients with ulcerative colitis were $2 \%$ by 10 years, $8 \%$ by 20 years, and $18 \%$ by 30 years of disease duration, respectively [7]. Jess et al. showed standardized incidence ratio of 1.9 for neoplasia in Crohn's disease [8]. Independent risk factors of carcinogenesis include young age at disease onset, more extensive colitis, greater inflammatory burden, concomitant primary sclerosing cholangitis, and a family history of colorectal cancer $[9,10]$.

The shift from IBD to colorectal cancer could be one salient example of the link between chronic inflammations and tumorigenesis since chronic inflammation is believed to have decisive roles in the pathogenesis of cancer [11, 12]. The molecular pathway leading to colorectal cancer in IBD appears to differ from the well-known sporadic cases. The pathological process of traditional sporadic colorectal cancer is "normal intestinal epithelium - adenoma adenocarcinoma". However, IBD associated tumorigenesis is characterized by an "inflammation - dysplasia - cancer" sequence in histopathology $[13,14]$. 
In order to study one certain disease, cell lines and animal models are usually essential tools. There are multitudinous cell lines for nearly all kinds of cancers. As to colorectal cancer, there exist many kinds of cell lines, such as HCT 116, HT 29, SW480 and RKO [15, 16]. And animal models of colorectal cancer are also mature, including subcutaneous injection with tumor cell in nude mice, injecting tumor cell directly on the bowel wall by surgery, or repeated injection with carcinogenic reagents $[17,18]$. As to IBD, no commercial cell lines is available, but some studies begin to focus on primary culture cell [19]. Our understanding of IBD depends mainly on rodent models. And animal model of IBD is often performed by adding pro-inflammatory reagent such as dextran sulfate sodium into the drinking water, or $\mathrm{T}$ cell transfer colitis in mice with severe combined immunodeficiency $[20,21]$. In fact, IBD associated tumorigenesis is a slow and dynamic disease process, from inflammation to tumorigenesis. And up to now, no cell model is available. In this study, a mice model is introduced, which mimics the disease course of IBD associated tumorigenesis.

\section{Materials and Methods}

\subsection{Mice}

Female C57BL/6 mice, aged 6-8 weeks were purchased from the Laboratory Animal Center of Sun Yat-sen University, Guangzhou, China. Mice were kept in special pathogen free facility with free access to drinking water and a pellet-based diet and were quarantined for 7 days before experiment. The experimental protocol was approved by the Ethical Committee of Sun Yat-sen University. All animal studies were conducted with the approval of the Institutional Animal Care and Use Committee of Sun Yat-sen University and also in compliance with the guidelines on animal welfare of the National Committee for Animal Experiments.

\subsection{Chemical Reagents}

Azoxymethane (AOM, Sigma-Aldrich, Saint Louis, USA), a downstream metabolite of dimethylhydrazine, is a chemical agent that can alkylate DNA, facilitate base mispairings and thus initiate cancer, especially in the colorectum.

Dextran sulfate sodium (DSS, 36-50 kDa, MP Biomedical, California, USA), a pro-inflammatory reagent, is especially toxic to the colon after dissolving in drinking water.

\subsection{Animal Model Induction Procedure}

Twelve female C57BL/6 mice (aged 6-8 weeks old, about $20 \mathrm{~g}$ ) were divided into 2 groups (6 mice per group), including the modeling group (receiving AOM/DSS modeling) and the control group (normal feeding, without AOM/DSS modeling). Briefly, the mice in the modeling group were injected intraperitoneally with a single dose $(10 \mathrm{mg} / \mathrm{kg})$ of AMO at the beginning of the experiment, followed by 3 cycles of DSS in drinking water, with each cycle consisting of 1 week of $2 \%$ DSS in the drinking water and 2 weeks of normal drinking water. After 3 cycles of DSS in drinking water, the mice were kept for another 3 weeks with normal drinking water. Procedure of modeling was shown in Figure 1.

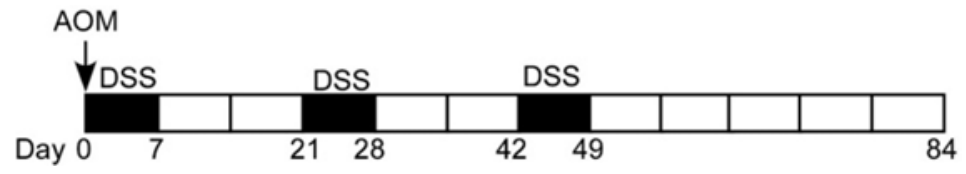

Figure 1. Schematic overview of model of IBD associated tumorigenesis induction by AOM/DSS.

Mice were monitored twice one week for the body weight, stool consistency and the presence of blood in the excreta. At the end of Week 12, mice in both groups were sacrificed by cervical dislocation. Length of colorectum (from the ileocecal junction to the anal verge) and spleen size were measured. Then colorectum was incised longitudinally and macroscopic tumors were counted and measured with a caliper. Segments of the distal colon and rectum were fixed in $10 \%$ neutral buffered formalin for subsequent paraffin embedding.

\subsection{Histopathological Evaluation}

Tissues from mice in the both groups were routinely sliced into 4 micrometer-thick after formalin fixing and paraffin embedding; and then the sections were stained with hematoxylin and eosin to evaluate the inflammation of the bowel and confirm the pathology of the tumors.

\subsection{Statistics Analysis}

Data were analyzed using the Statistical Package for Social Science software version 17.0 (SPSS Inc. Chicago, IL, USA). Values were expressed as mean \pm standard error mean (SEM) and analyzed with the Student's t-test. Differences with two-sided $\mathrm{p}$ values $<0.05$ were considered statistically significant.

\section{Results}

All the mice in both groups successfully survived till the end of the modeling procedure, but the mice in the modeling had some signs of illness, such as diarrhea with bloody stools and emaciation (Figure 2). Body weight loss was obvious in the modeling group and body weight had significant difference in the two groups $(\mathrm{P}<0.001$, shown in Figure 3$)$. 

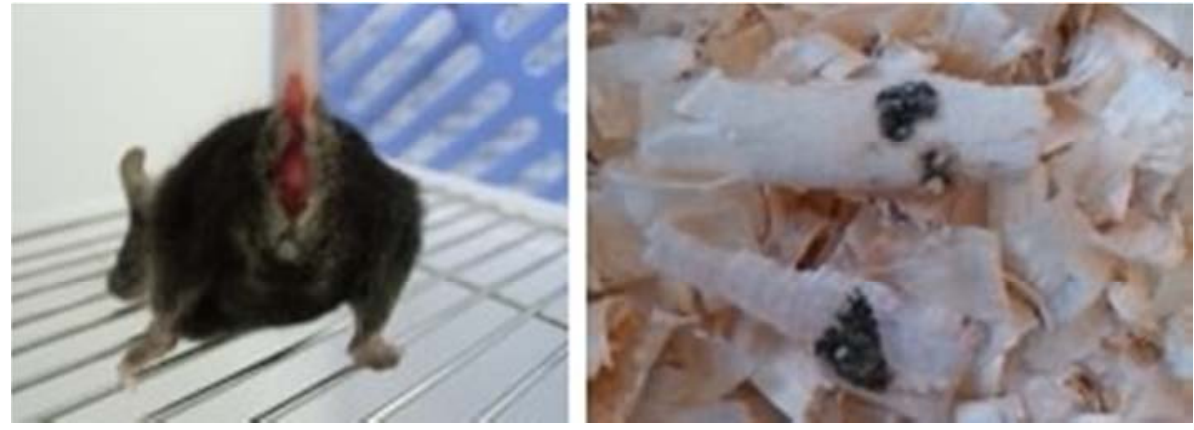

Figure 2. Mouse with bloody stools.

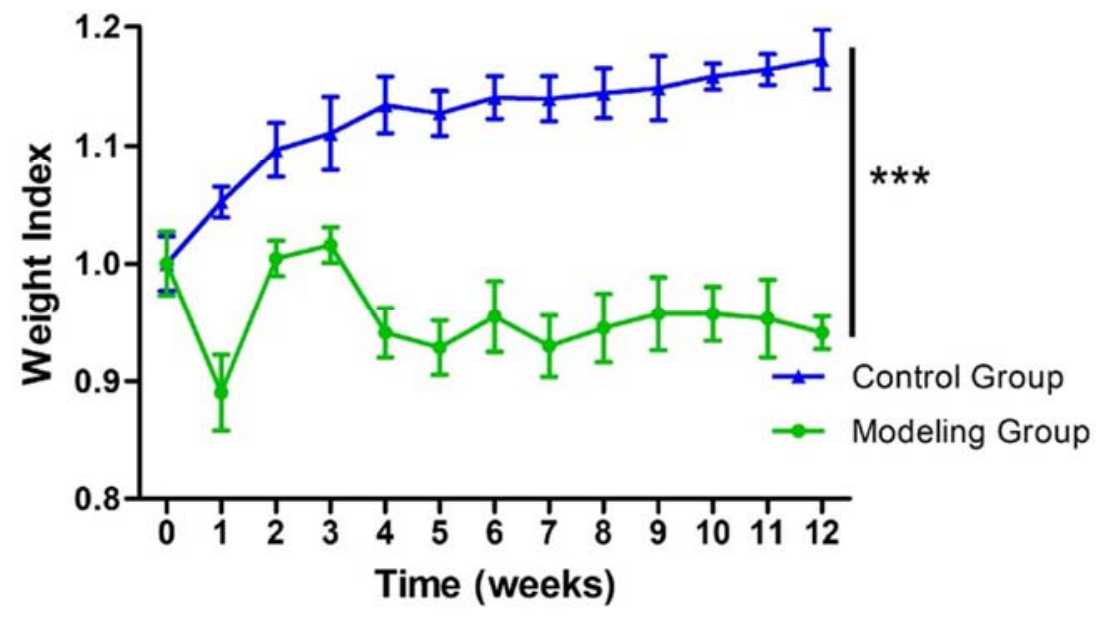

Figure 3. Changes in body weight. $* * * P<0.001$.

On the 84th day, mice were sacrificed and dissected. The colorectum was obvious swollen in the modeling group. By measure without tension, the length of colorectum in the modeling group was significantly shorter compared with the

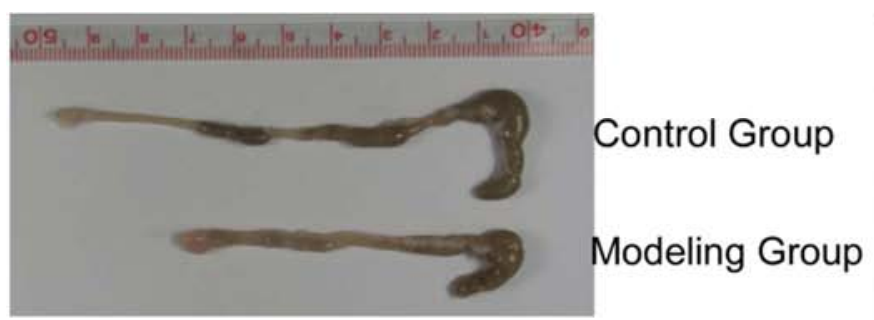

control group $(\mathrm{P}<0.001)$. On the contrary, the size of the spleen in the modeling was bigger $(\mathrm{P}<0.001)$. The comparision of colorectum and spleen was shown in Figure 4.
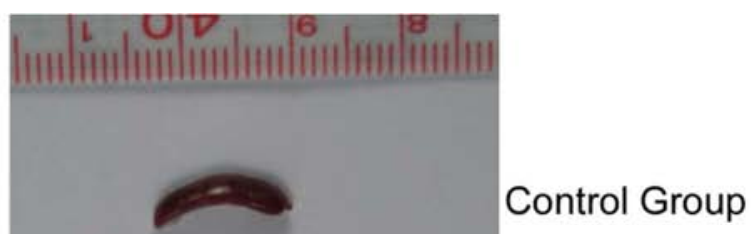

Modeling Group
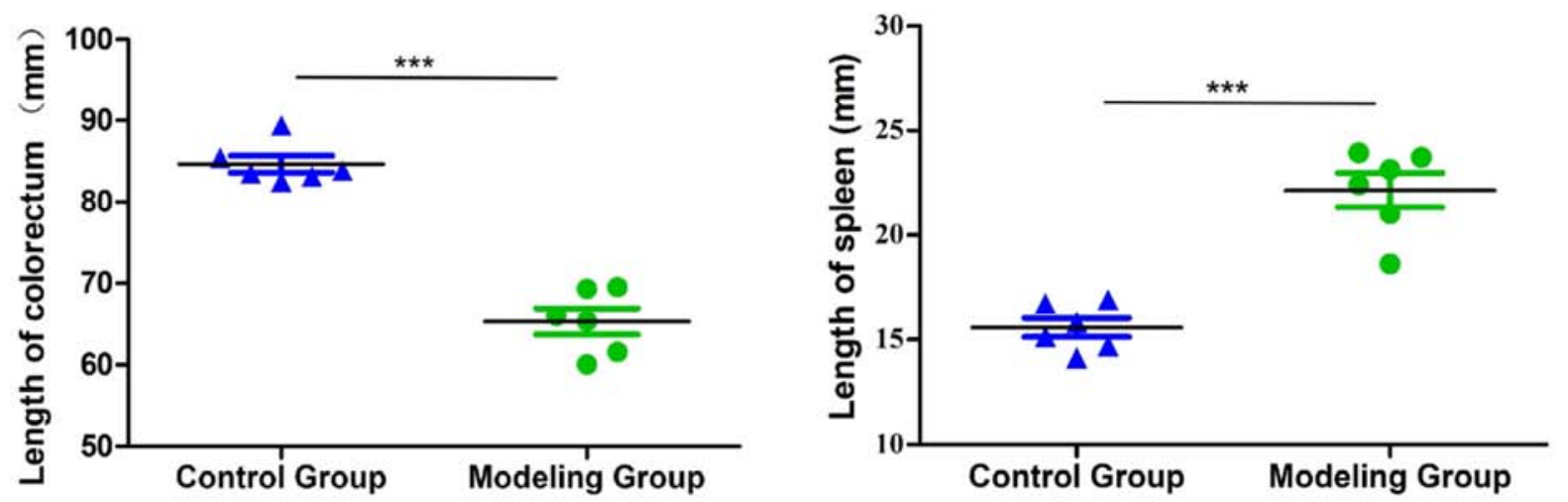

Figure 4. Comparison of length of colorectum (left) and spleen (right). $* * * P<0.001$. 
Then the colorectum was incised lengthways along the mesangial side, and tumors were observed in the modeling group, mainly between the mid colon and the distal rectum (shown in Figure 5). There were averagely 6.5 tumors in the colorectum in the modeling group with an average tumor size of $10.25 \mathrm{~mm}^{3}$.

\section{A}

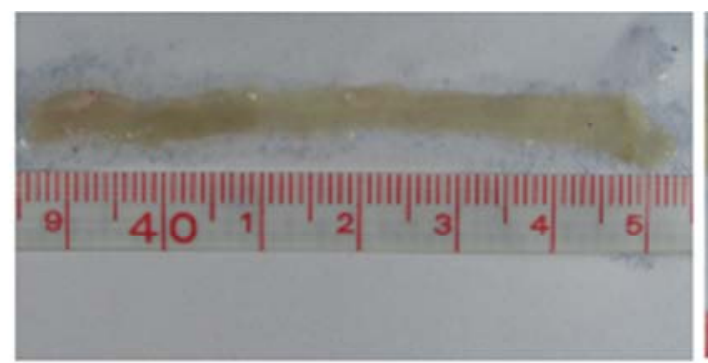

B

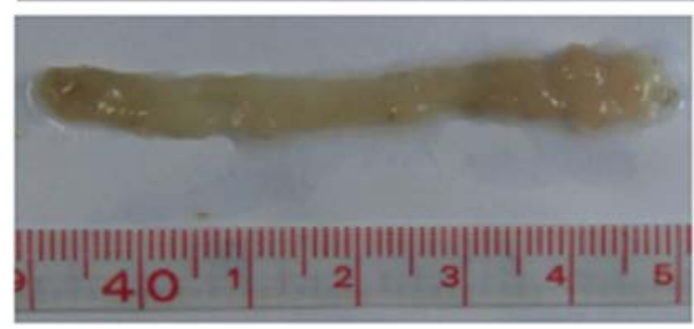

After general observation, colorectum tissue was stained with hematoxylin and eosin, histological damage with inflammation was found and all tumors in the modeling group were characterized as adenomas with high-grade dysplasia (shown in Figure 6).
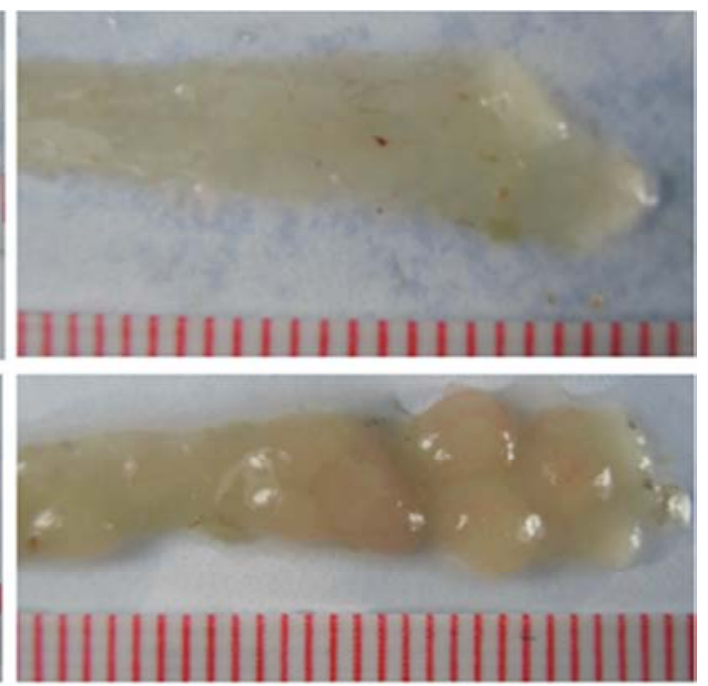

Figure 5. Colorectum of mice in the control group (A) and the modeling group (B). Tumors were observed on the colorectum in mice in the modeling group.

A

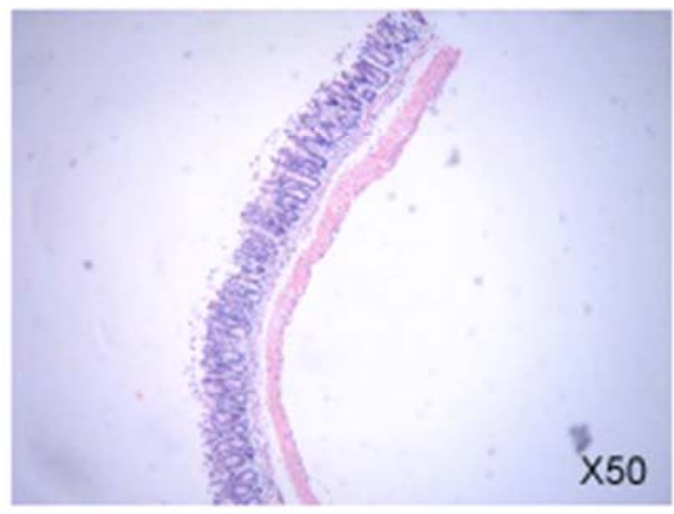

B

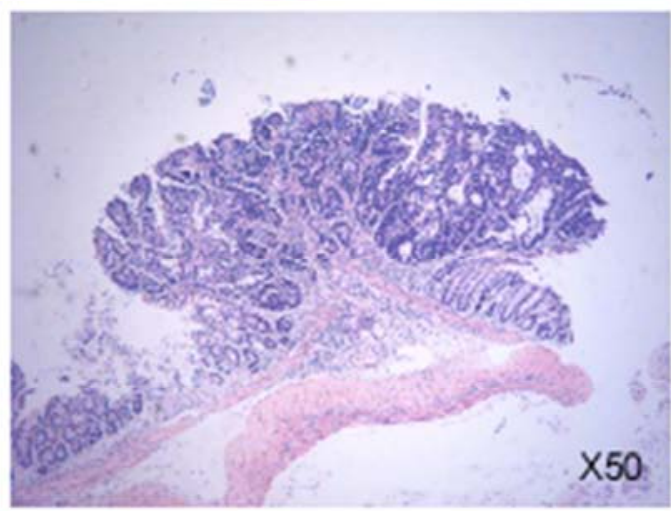

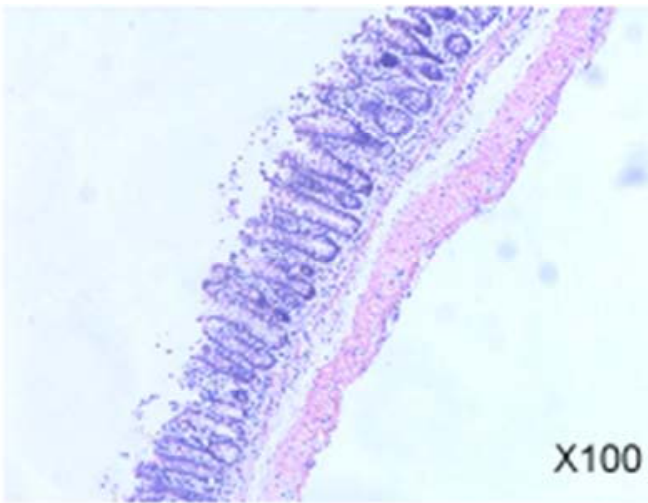

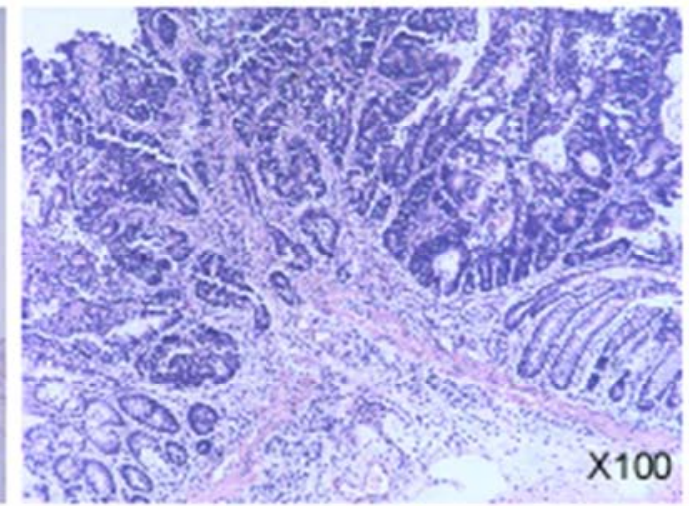

Figure 6. Histopathological evaluation for the colorectum in the control group (A) and the modeling group (B).

\section{Conclusion}

In this study, a mice model mimicking the dynamic disease course of IBD associated tumorigenesis is built. The model is characterized by swollen and shorten bowel with tumors in the colorectum. It is an inducible and stable model, and would be very useful in related research in this field such as pathological process, prevention or treatment of IBD associated tumorigenesis. 


\section{References}

[1] Endo K, Shiga H, Kinouchi Y, Shimosegawa T. Inflammatory bowel disease: IBD. Rinsho Byori, 2009, 57(6): 527-532.

[2] Soderlund S, Brandt L, Lapidus A, Karlen P, Brostrom O, Lofberg R, et al. Decreasing time-trends of colorectal cancer in a large cohort of patients with inflammatory bowel disease. Gastroenterology, 2009, 136(5): 1561-1567, 1818-1819.

[3] Adami H O, Bretthauer M, Emilsson L, Hernan M A, Kalager $\mathrm{M}$, Ludvigsson $\mathrm{J} \mathrm{F}$, et al. The continuing uncertainty about cancer risk in inflammatory bowel disease. Gut, 2016, 65(6): 889-893.

[4] Itzkowitz S H, Harpaz N. Diagnosis and management of dysplasia in patients with inflammatory bowel diseases. Gastroenterology, 2004, 126(6): 1634-1648.

[5] Vagefi P A, Longo W E. Colorectal cancer in patients with inflammatory bowel disease. Clin Colorectal Cancer, 2005, 4(5): 313-319.

[6] Herszenyi L, Miheller P, Tulassay Z. Carcinogenesis in inflammatory bowel disease. Dig Dis, 2007, 25(3): 267-269.

[7] Eaden J A, Abrams K R, Mayberry J F. The risk of colorectal cancer in ulcerative colitis: a meta-analysis. Gut, 2001, 48(4): 526-535.

[8] Jess T, Gamborg M, Matzen P, Munkholm P, Sorensen T I. Increased risk of intestinal cancer in Crohn's disease: a meta-analysis of population-based cohort studies. Am J Gastroenterol, 2005, 100(12): 2724-2729.

[9] Lakatos L, Mester G, Erdelyi Z, David G, Pandur T, Balogh M, et al. Risk factors for ulcerative colitis-associated colorectal cancer in a Hungarian cohort of patients with ulcerative colitis: results of a population-based study. Inflamm Bowel Dis, 2006, 12(3): 205-211.

[10] Loftus E J. Epidemiology and risk factors for colorectal dysplasia and cancer in ulcerative colitis. Gastroenterol Clin North Am, 2006, 35(3): 517-531.

[11] Grivennikov S I, Greten F R, Karin M. Immunity, inflammation, and cancer. Cell, 2010, 140(6): 883-899.

[12] Fitzpatrick F A. Inflammation, carcinogenesis and cancer. Int Immunopharmacol, 2001, 1(9-10): 1651-1667.

[13] Ullman T A, Itzkowitz S H. Intestinal inflammation and cancer. Gastroenterology, 2011, 140(6): 1807-1816.

[14] Baker A M, Cross W, Curtius K, Al B I, Choi C R, Davis H L, et al. Evolutionary history of human colitis-associated colorectal cancer. Gut, 2018.

[15] Liu Y, Lu Y, Zhou M, Zhang C, Li X. Construction of colorectal cancer cell line stably expressing mir-101 and identification of the target gene of mir-101. Nan Fang Yi Ke Da Xue Xue Bao, 2014, 34(7): 928-933.

[16] Mathieu A A, Ohl-Seguy E, Dubois M L, Jean D, Jones C, Boudreau F, et al. Subcellular proteomics analysis of different stages of colorectal cancer cell lines. Proteomics, 2016, 16(23): 3009-3018.

[17] Boivin G P, Washington K, Yang K, Ward J M, Pretlow T P, Russell R, et al. Pathology of mouse models of intestinal cancer: consensus report and recommendations. Gastroenterology, 2003, 124(3): 762-777.

[18] Day C P, Merlino G, Van Dyke T. Preclinical mouse cancer models: a maze of opportunities and challenges. Cell, 2015, 163(1): 39-53.

[19] Dotti I, Salas A. Potential Use of Human Stem Cell-Derived Intestinal Organoids to Study Inflammatory Bowel Diseases. Inflamm Bowel Dis, 2018.

[20] Manicassamy S, Manoharan I. Mouse models of acute and chronic colitis. Methods Mol Biol, 2014, 1194: 437-448.

[21] Ostanin D V, Bao J, Koboziev I, Gray L, Robinson-Jackson S A, Kosloski-Davidson M, et al. T cell transfer model of chronic colitis: concepts, considerations, and tricks of the trade. Am J Physiol Gastrointest Liver Physiol, 2009, 296(2): G135-G146. 